\title{
Физиологические показатели
}

\section{при различном прогнозе острого периода геморрагического инсульта}

\author{
И.С. Курепина ${ }^{1}$, Р.А. Зорин ${ }^{1}$, А.А. Косолапов ${ }^{2}$ \\ ${ }^{1}$ ФГБоу ВО «Рязанский государственный медицинский университет имени академика И.П. Павлова» Министерства \\ здравоохранения Российской Федерации; Россия, г. Рязань
}

2 ГБУ РО «Областная клиническая больница»; Россия, г. Рязань

\section{РЕЗЮМЕ}

\begin{abstract}
Целью исследования является выявление различий физиологических показателей в группах пациентов в остром периоде геморрагического паренхиматозного инсульта (супратенториальных полушарных гематом) с благоприятным прогнозом и летальным исходом. Дизайн: проспективное исследование.

Материалы и методы. Обследованы 96 пациентов, страдающих геморрагическим инсультом супратенториальной локализации. Диагноз геморрагического инсульта определялся на основе данных нейровизуализации, клинической синдромологии и анамнеза, клинико-лабораторных данных. На основе кластерного анализа и экспертных оценок выделены две группы: 49 пациентов с летальным исходом острого периода геморрагического инсульта и 47 человек с благоприятным прогнозом. Проводилась оценка уровня сознания, когнитивных функций, нейрофизиологических показателей: электроэнцефалография (ЭЭГ), исследование когнитивных вызванных потенциалов Р300, вариабельности сердечного ритма (ВСР).

Результаты. У больных с благоприятным прогнозом полная амплитуда спектра ЭЭГ была выше в сравнении с таковой у лиц с летальным исходом (статистически значимые результаты представлены во всех отведениях). Выявлено значимое увеличение средней частоты тета-колебаний в обеих группах, особенно в группе летального исхода. При исследовании Р300 у пациентов с летальным исходом заболевания зафиксирована значимо меньшая амплитуда P2N2 в Fz, чем при благоприятном прогнозе: 5,1 (2,6; 9,1) мкB против 8,9 (5,6; $20,4)$ мкB $(U=148 ; p=0,021)$. При сравнительном анализе BCP значимые различия выявлены только по частоте сердечных сокращений (среднему интервалу R-R): она была ниже у больных с летальным исходом острого периода геморрагического инсульта: 696 (608; 836) мс против 806 (743; 911) мс $(U=181 ; p=0,033)$. У пациентов с летальным исходом определялось увеличение числа линейных корреляций физиологических показателей по сравнению с таковым у больных с благоприятным прогнозом.

Заключение. Летальному исходу острого периода геморрагического инсульта предшествуют редукция основного коркового ритма, нарастание медленно-волновой активности, снижение активации механизмов опознания стимула, по данным когнитивных вызванных потенциалов Р300. Анализ степени сопряжения физиологических механизмов регуляции уровня функциональной активности головного мозга (ЭЭГ), нейрофизиологических коррелятов опознания стимула и принятия решения по отношению к нему (Р300), а также механизмов вегетативной регуляции (ВСР) указывает на ограничение функциональных резервов у больных с летальным исходом заболевания. Ключевые слова: геморрагический инсульт, потенциал, связанный с событием, вариабельность сердечного ритма, корреляционный анализ.
\end{abstract}

Вклад авторов: Курепина И.С., Косолапов А.А. - отбор, обследование пациентов, обзор публикаций по теме статьи, статистическая обработка данных, написание текста рукописи; Зорин Р.А. - анализ и интерпретация данных, разработка дизайна исследования, проверка критически важного содержания, утверждение рукописи для публикации.

Конфликт интересов: авторы заявляют об отсутствии возможных конфликтов интересов.

Для цитирования: Курепина И.С., Зорин Р.А., Косолапов А.А. Физиологические показатели при различном прогнозе острого периода геморрагического инсульта. Доктор.Ру. 2021; 20(9): 11-16. DOI: 10.31550/1727-2378-2021-20-9-11-16

\section{Physiological Parameters in Various Prognosis of the Acute Period of Haemorrhagic Stroke}

\author{
I.S. Kurepina ${ }^{1}$, R.A. Zorin ${ }^{1}$, A.A. Kosolapov ${ }^{2}$ \\ ${ }^{1}$ I.P. Pavlov Ryazan State Medical University of the Ministry of Health of the Russian Federation; 9 Vysokovoltnaya Str., Ryazan, Russian \\ Federation 390026 \\ ${ }^{2}$ Regional Clinical Hospital; $3 a$ Internatsionalnaya Str., Ryazan, Russian Federation 390039
}

\section{ABSTRACT}

Study Objective: To identify the differences in physiological parameters in groups of patients in an acute period of haemorrhagic parenchymatous stroke (supratentorial hemisphere hematomas) with favourable outcome and fatality.

Study Design: prospective study.

Materials and Methods. We have examined 96 patients with supratentorial haemorrhagic stroke. Haemorrhagic stroke was diagnosed on the basis of neuroimaging results, clinical symptoms and medical history, as well as clinical and laboratory data. A cluster analysis and expert

Курепина Инна Сергеевна (автор для переписки) - аспирант кафедры неврологии и нейрохирургии ФГБоу ВО «РязГму им. акад. П.П. Павлова» Минздрава России. 390026, Россия, г. Рязань, ул. Высоковольтная, д. 9. eLIBRARY.RU SPIN: 6914-4106. https://orcid.org/00000001-9207-2447. E-mail: innakurepina90@mail.ru

(Окончание на с. 12.) 
examinations made it possible to divide patients into two groups: 49 patients who died of the acute haemorrhagic stroke and 47 patients with a favourable outcome. We evaluated the level of consciousness, cognitive functions, neurophysiological parameters: electroencephalography (EEG), cognitive evoked potentials P300, heart rate variability (HRV).

Study Results. In patients with a favourable outcome, an overall amplitude of the EEG spectrum was higher vs. patients with lethal outcome (statistically significant results were noted in all leads). A significant increase in the mean frequency of theta waves was seen in both groups, especially in the group with lethal outcomes. When P300 in patients with lethal outcome was evaluated, a significantly lower P2N2 amplitude in Fz was noted vs favourable outcome group: $5.1(2.6 ; 9.1) \mu \mathrm{V} v s 8.9(5.6 ; 20.4) \mu \mathrm{V}(\mathrm{U}=148 ; \mathrm{p}=0.021)$. A comparative analysis of HRV revealed significant differences only in heart rate (mean $\mathrm{R}-\mathrm{R})$ : it was lower in patients who died in the acute period of haemorrhagic stroke: $696(608 ; 836) \mathrm{ms}$ vs $806(743 ; 911) \mathrm{ms}(\mathrm{U}=181 ; \mathrm{p}=0.033)$. In patients with lethal outcomes, there is an increase in the number of linear correlations in physiological parameters vs. favourable outcome group.

Conclusion. Deaths from acute haemorrhagic stroke are preceded by reduction in the basic cortical rhythm, growth in slow waves activity, reduction in the stimuli recognition mechanism activation (according to cognitive evoked potentials P300). An analysis of the rate of correlation in physiological mechanisms of brain activity regulation (EEG), neurophysiological correlates in stimuli recognition and decisionmaking (P300), as well as autonomic regulation mechanisms (HRV) shows limited functional reserves in patients with lethal outcomes.

Keywords: haemorrhagic stroke, event-associated potential, heart rate variability, correlation analysis.

Contributions: Kurepina, I.S. and Kosolapov, A.A. - patient selection and examination, review of thematic publications, statistical data processing, text of the article; Zorin, R.A. - data analysis and interpretation, study design, review of critically important material, approval of the manuscript for publication.

Conflict of interest: The authors declare that they do not have any conflict of interests.

For citation: Kurepina I.S., Zorin R.A., Kosolapov A.A. Physiological Parameters in Various Prognosis of the Acute Period of Haemorrhagic Stroke. Doctor.Ru. 2021; 20(9): 11-16. (in Russian). D0I: 10.31550/1727-2378-2021-20-9-11-16

\section{ВВЕДЕНИЕ}

Геморрагический инсульт является вторым по распространенности типом инсульта, что составляет 10-50\% случаев в зависимости от населения, расы и региона. Диапазон летальности - от $35 \%$ через 7 дней до 59\% через год; $50 \%$ смертельных случаев происходят в первые 48 часов после появления первых клинических симптомов $[1,2]$. Оставшиеся в живых часто остаются с тяжелой инвалидностью, и лишь менее 40\% пациентов восстанавливают функциональную независимость [3].

Оценка нейрофизиологических показателей в остром периоде геморрагического инсульта позволяет использовать дополнительные предикторы дальнейшего течения заболевания наряду с клиническими и нейровизуализационными данными [4-6].

В целом худший прогноз ассоциирован с замедлением или редукцией альфа-ритма как основного коркового ритма, нарастанием индекса и мощности медленно-волновой активности, избыточной активацией симпатического контура автономной нервной системы. Исследование когнитивных вызванных потенциалов позволяет дополнительно оценить нейрофизиологические корреляты степени выраженности расстройства сознания $[7,8]$.

Целью исследования является выявление различий физиологических показателей в группах пациентов в остром периоде геморрагического паренхиматозного инсульта (супратенториальных полушарных гематом) с благоприятным прогнозом и летальным исходом.

\section{МАТЕРИАЛЫ И МЕТОДЫ}

С целью изучения исходной сопоставимости групп проанализированы анамнестические данные, показатели клинического, инструментального и лабораторного обследований 96 пациентов. Исследование проводилось в 0бластной клинической больнице Рязани с 2018 по 2021 г. Среди участников было несколько больше мужчин ( $\mathrm{n}=52 ; 54,2 \%)$. Сопоставление больных по возрасту выявило преобладание возрастной категории 50-59 лет среди мужчин $(\mathrm{n}=16 ; 30,8 \%)$ и 70-79 лет среди женщин $(\mathrm{n}=12 ; 27,3 \%)$. Средний возраст женщин составил 73,5 года, мужчин - 66,1 года.

Рентгеновская КТ выполнялась на аппарате Toshiba Aquilion 64 (Toshiba, Япония).

Диагноз геморрагического инсульта определялся на основе данных нейровизуализации, клинической синдромологии и анамнеза (особенностей начала и течения заболевания), клинико-лабораторных данных.

Критерии включения в исследование:

1) нетравматическая полушарная гематома супратенториальной локализации, верифицированная в день поступления, что подтверждено рентгеновской КТ;

2) отсутствие у пациентов показаний для хирургического лечения.

Критерии исключения: субарахноидальные кровоизлияния, кровоизлияния аневризматического характера, только вентрикулярные кровоизлияния.

Для оценки динамики состояния пациентов с геморрагическим инсультом определяли показатели Cerebrolysin and recovery after stroke, а также шкалы комы Глазго на 1-е (день поступления), 3-и и 21-е сутки.

На основе кластерного анализа и экспертных оценок выделены две группы: 49 пациентов с летальным исходом острого периода геморрагического инсульта и 47 человек с благоприятным прогнозом.

Первично выделение группы пациентов с летальным исходом основывалось на их гибели вследствие отека-набухания головного мозга.

Регистрация ЭЭГ осуществлялась при помощи программного комплекса «Нейрон-Спектр.Net» по схеме 10-20 при 19-канальной записи с анализом спектральных показателей ЭЭГ в парадигме oddball (при невозможности реакции пациента

Зорин Роман Александрович - д. м. н., доцент кафедры неврологии и нейрохирургии ФГБоУ ВО «РязГМУ им. акад. П.П. Павлова» Минздрава России. 390026, Россия, г. Рязань, ул. Высоковольтная, д. 9. eLIBRARY.RU SPIN: 5210-5747. https://orcid.org/0000-0003-4310-8786. E-mail: zorin.ra30091980@mail.ru

Косолапов Андрей Алексеевич - к. м. н., заведующий нейрохирургическим отделением ГБУ РО ОКБ. 390039, Россия, г. Рязань, ул. Интернациональная, д. 3a. http://orcid.org/0000-0001-7593-2353. E-mail: kocolapov2@rambler.ru

(Окончание. Начало см. на с. 11.) 
на стимул производилась подача различающихся стимулов без нажатия на кнопку). Потенциал Р300 регистрировали в рамках вероятностной парадигмы появления значимого стимула (тон 2000 Гц) и незначимого стимула (тон 1000 Гц). Вероятность появления значимого стимула составила $30 \%$, незначимого 70\%, длительность стимула была 50 мс интенсивность - 70 Дб SPL, использовались наушники $[9,10]$.

Для регистрации вариабельности сердечного ритма (ВСР) применялись прибор «Варикард 2.5» (фирма «Рамена», Рязань) и программа ИСКИМ (версия 6.0). Оценивались ЧСС, среднее квадратичное отклонение, коэффициент вариации, индекс напряжения регуляторных систем.

Выполнен анализ мощности спектральных составляющих динамического ряда кардиоинтервалов с определением мощности медленных волн 1-го (LF) и 2-го порядка (VLF) [11].

Оценка статистической значимости различий между группами проводилась методами непараметрической статистики с применением критерия Манна - Уитни U (Z), описание данных предполагало выделение медианы (Me), нижнего (LQ) и верхнего квартилей (UQ). Искусственные нейронные сети создавались, обучались и тестировались при помощи программы Statistica 10.0 Ru.

В качестве контрольной группы (для оценки валидности используемых нейрофизиологических показателей) обследованы 28 пациентов с дорсопатией шейного отдела позвоночника (умеренным мышечно-тоническим синдромом) вне стадии обострения и без неврологических нарушений, указаний на эпизоды нарушения мозгового кровообращения в анамнезе и признаков компрессии и гемодинамически значимых стенозов магистральных сосудов шеи, по данным ультразвуковой доплерографии.

Потенциал, связанный с событием: интенсивность обоих видов стимулов - порядка 60-80 дБ (иногда до 100 дБ); частота низкого тона - 1000 Гц, высокого - 2000 Гц. Появление значимого стимула выставляется с вероятностью 20-30\% от общего количества стимулов. Длительность стимула - 50 мс. Межимпульсный интервал - 1-2 с.

\section{РЕЗУЛЬТАТЫ И ОБСУЖДЕНИЕ}

В таблице 1 представлены показатели частоты альфа-колебаний в выделенных группах.

Паттерны спонтанной ЭЭГ значимо не различались в параметрах распределения ритмизированной альфа-активности по уровню амплитуды. В группе с летальным исходом фоновая активность была представлена так называемым медлен- ным вариантом альфа-ритма. В группе с благоприятным прогнозом доминирующая активности составила 10,1-10,5 Гц против 10,0-10,3 Гц в группе летального исхода.

Генерация альфа-ритма связана с реверберацией импульсной активности по интеркортикальным и таламо-кортикальным нейронным сетям, а выраженность обусловливает синхронизацию функциональной активности различных мозговых систем, например определяет связь получаемой от афферентной системы организма информации с механизмами оперативной памяти, регулируя адаптационные процессы.

В таблице 2 представлены показатели амплитуды альфа-колебаний.

у больных с благоприятным прогнозом полная амплитуда спектра ЭЭГ была выше в сравнении с таковой у лиц с летальным исходом (статистически значимые результаты представлены во всех отведениях).

Выявлено значимое увеличение средней частоты тета-колебаний в обеих группах, особенно в группе летального исхода (табл. 3).

Таким образом, данные анализа ЭЭГ отражают редукцию основного коркового ритма, увеличение синхронизирующих стволовых влияний с нарастанием медленно-волновой активности, предшествующие летальном исходу геморрагического инсульта.

При исследовании Р300 у пациентов с летальном исходом заболевания выявлена значимо меньшая амплитуда P2N2 B Fz, чем при благоприятном прогнозе: 5,1 (2,6; 9,1) мкB против 8,9 $(5,6 ; 20,4)$ мкВ $(U=148 ; p=0,021)$.

По мнению некоторых авторов, генерируют компонент Р300 таламус, гиппокамп, лобные доли, теменная область коры головного мозга, подкорковые структуры [12, 13]. Предполагают, что компонент N2 (негативная фаза) отражает процессы, происходящие в височной области. У наших пациентов с благоприятным прогнозом можно сделать вывод о более высокой активации лобных долей по сравнению с показателем больных из группы летального исхода.

При сравнительном анализе ВСР значимые различия выявлены только по ЧСС (среднему интервалу R-R): она была ниже в группе пациентов с летальным исходом острого периода геморрагического инсульта: $696(608 ; 836)$ мс против $806(743 ; 911)$ мс $(U=181 ; p=0,033)$.

Существенные различия по другим показателям ВСР отсутствовали. Однако параметры ВСР применены нами для определения взаимосвязи между физиологическими показателями методом корреляционного анализа (рис. 1,2).

\section{Таблица 1 / Table 1}

Частота альфа-колебаний в группах с благоприятным прогнозом и Аетальным исходом инсульта, Гц

Alfa waves in groups with favourable and lethal outcomes of the stroke, $\mathrm{Hz}$

\begin{tabular}{|c|c|c|c|c|c|c|c|c|}
\hline \multirow[t]{2}{*}{ Отведения / Leads } & \multicolumn{3}{|c|}{$\begin{array}{c}\text { Пациенты с благоприятным } \\
\text { прогнозом / Patients with } \\
\text { favourable outcome }\end{array}$} & \multicolumn{3}{|c|}{$\begin{array}{l}\text { Пациенты с летальным исходом / } \\
\text { Patients with lethal outcome }\end{array}$} & \multirow[t]{2}{*}{ U } & \multirow[t]{2}{*}{$\mathbf{P}$} \\
\hline & $\mathrm{Me}$ & LQ & UQ & $\mathrm{Me}$ & LQ & UQ & & \\
\hline T3 & 10,3 & 10,0 & 10,5 & 10,1 & 9,8 & 10,5 & 329 & 0,049 \\
\hline $\mathrm{T} 4$ & 10,2 & 10,1 & 10,6 & 10,2 & 9,7 & 10,5 & 379 & 0,207 \\
\hline P3 & 10,3 & 10,1 & 10,6 & 9,9 & 9,7 & 10,5 & 315 & 0,030 \\
\hline P4 & 10,2 & 10,0 & 10,5 & 10,0 & 9,7 & 10,4 & 337 & 0,063 \\
\hline 01 & 10,3 & 10,0 & 10,6 & 10,1 & 9,7 & 10,5 & 662 & 0,059 \\
\hline 02 & 10,2 & 10,1 & 10,5 & 10,0 & 9,7 & 10,3 & 656 & 0,025 \\
\hline
\end{tabular}


Амплитуда альфа-колебаний в выделенных группах с благоприятным прогнозом и метальным исходом инсульта, мкВ

Alfa waves amplitude in groups with favourable and lethal outcomes of the stroke, $\mu \mathrm{V}$

\begin{tabular}{|c|c|c|c|c|c|c|c|c|}
\hline \multirow[t]{2}{*}{ Отведения / Leads } & \multicolumn{3}{|c|}{$\begin{array}{c}\text { Пациенты с благоприятным } \\
\text { прогнозом / Patients with } \\
\text { favourable outcome }\end{array}$} & \multicolumn{3}{|c|}{$\begin{array}{c}\text { Пациенты с летальным исходом / } \\
\text { Patients with lethal outcome }\end{array}$} & \multirow[t]{2}{*}{$\mathbf{U}$} & \multirow[t]{2}{*}{$\mathbf{P}$} \\
\hline & Me & LQ & UQ & Me & LQ & UQ & & \\
\hline F3 & 22 & 17 & 26 & 16 & 12 & 22 & 666 & 0,002 \\
\hline F4 & 24 & 20 & 28 & 19 & 14 & 23 & 704 & 0,006 \\
\hline P3 & 25 & 21 & 30 & 18 & 14 & 23 & 651 & 0,001 \\
\hline P4 & 26 & 23 & 32 & 20 & 16 & 24 & 635 & 0,001 \\
\hline T3 & 25 & 21 & 30 & 18 & 14 & 23 & 607 & 0,001 \\
\hline T4 & 27 & 23 & 33 & 20 & 16 & 25 & 616 & 0,001 \\
\hline
\end{tabular}

Мощность тета-колебаний эмектроэнцефалограммы в группах с благоприятным прогнозом и метальным исходом инсульта в фоновом режиме, мкВ² $/ \mathrm{c}^{2}$

Background theta waves on an electroencephalogram in groups with favourable and lethal outcomes of the stroke, $\mu \mathrm{V}^{2} / \mathrm{s}^{2}$

\begin{tabular}{|c|c|c|c|c|c|c|c|c|}
\hline \multirow[t]{2}{*}{ Отведения / Leads } & \multicolumn{3}{|c|}{$\begin{array}{c}\text { Пациенты с благоприятным } \\
\text { прогнозом / Patients with } \\
\text { favourable outcome }\end{array}$} & \multicolumn{3}{|c|}{$\begin{array}{l}\text { Пациенты с летальным исходом / } \\
\text { Patients with lethal outcome }\end{array}$} & \multirow[t]{2}{*}{$\mathbf{U}$} & \multirow[t]{2}{*}{$\mathbf{P}$} \\
\hline & Me & LQ & UQ & $\mathrm{Me}$ & LQ & UQ & & \\
\hline F3 & 15,2 & 5,3 & 26,7 & 26,2 & 12,3 & 60,0 & 334 & 0,058 \\
\hline $\mathrm{F} 4$ & 18,1 & 6,9 & 28,4 & 26,3 & 9,8 & 81,8 & 302 & 0,018 \\
\hline T3 & 12,4 & 6,2 & 22,5 & 22,8 & 9,1 & 51,7 & 333 & 0,057 \\
\hline $\mathrm{T} 4$ & 11,4 & 6,7 & 22,6 & 17,3 & 7,0 & 44,3 & 333 & 0,057 \\
\hline P3 & 14,1 & 6,3 & 25,6 & 25,1 & 9,2 & 59,6 & 352 & 0,102 \\
\hline $\mathrm{P} 4$ & 11,1 & 7,9 & 22,5 & 26,8 & 5,5 & 57,7 & 346 & 0,085 \\
\hline
\end{tabular}

Рис. 1. Графическое отражение (корреляционная плеяда) минейных корреляций (Rs) физиологических показателей в группе пациентов с благоприятным прогнозом острого периода геморрагического инсульта: сплошные Аинии — положительные корреляции, штриховая Аиния - отрицательная корреляция

Fig. 1. Graphical representation (correlation pleiad) of linear correlations (Rs) of physiological parameters in the group of patients with favourable outcome in the acute period of haemorrhagic stroke: solid lines - positive correlations, dotted lines negative correlation

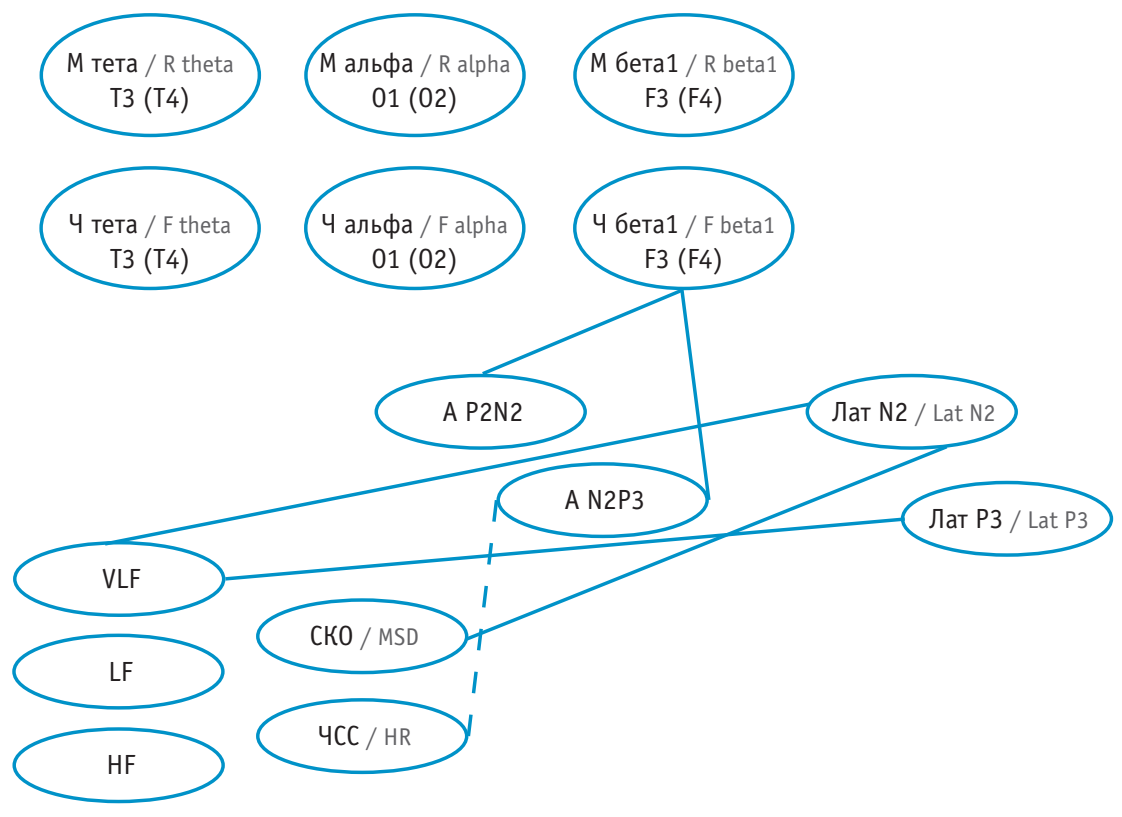


Рис. 2. Графическое отражение (корреляционная плеяла) минейных корреляций (Rs) физиологических показателей в группе пациентов с метальным исходом острого периода геморрагического инсульта: сплошные Аинии - положительные корреляции, штриховые Аинии - отрицательные корреляции; толщина Аиний отражает силу корреляций (тонкая Аиния - слабая корреляция).

Примечание. Здесь и в рисунке 1: $М$ тета Т3 (Т4) - мочность тета-колебаний электроэнчефалограммь (ЭЭГ) в отведении Т3 (или Т4), М альфа О1 (О2) — мочность альфа-колебаний ЭЭГ в отведении $О 1$ (или О2), М бета1 F3 (F4) - моцность бета1-колебаний ЭЭГ в отведении F3 (или F4), Ч тета Т3 (T4) — частота тета-колебаний ЭЭГ в отведении Т3 (или Т4), Ч альфа О1 (O2) — частота альфа-колебаний ЭЭГ в отведении О1 (или О2), Ч бета1 F3(F4) — частота бета1-колебаний ЭЭГ в отведении F3 (или F4), A P2N2 — амплитуда P2N2 P300 (F₹, C;

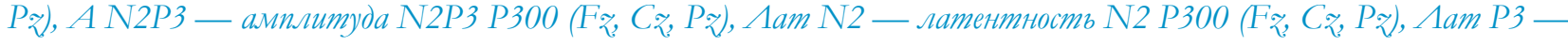

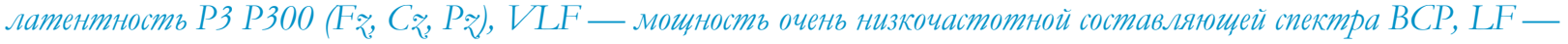
мочность низкочастотной составляншей спектра ВСР, НF — мочность высокочастотной составлятощей спектра ВСР, СКО — среднее квадратичное отклонение ВСР, ЧСС — частота сердечныгх сокращений

Fig. 2. Graphical representation (correlation pleiad) of linear correlations (Rs) of physiological parameters in the group of patients with lethal outcome in the acute period of haemorrhagic stroke: solid lines - positive correlations, dotted lines negative correlation; line thickness corresponds to the rate of correlation (thin line = poor correlation).

Note. Legend for Fig. 1 and Fig. 2: R theta T3 (T4) - theta wave rate on an electroencephalogram (EEG) in T3 (or T4) lead; R alpha O1 (O2) alpha wave rate on EEG in $\mathrm{O} 1$ (or $\mathrm{O} 2$ ) lead; $\mathrm{R}$ beta1 $\mathrm{F} 3$ ( F4) - beta1 wave rate on EEG in F3 (or F4) lead; F theta T3 (T4) - frequency of theta waves on EEG in T3 (or T4) lead; F alpha O1 (O2) - frequency of alpha waves on EEG in O1 (or O2) lead; F beta1 F3 (F4) frequency of beta1 waves on EEG in F3 (or F4) lead; A P2N2 - P2N2 P300 amplitude( F\%, C₹, Pr); A N2P3 - N2P3 P300 amplitude

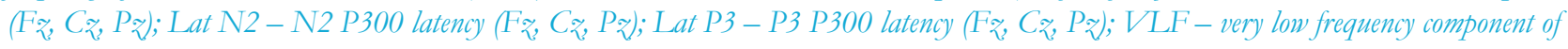
HRV spectrum; LF - low frequency component of HRV spectrum; HF - bigh frequency component of HRV; MSD - mean square deviation of HRV; HR - beart rate

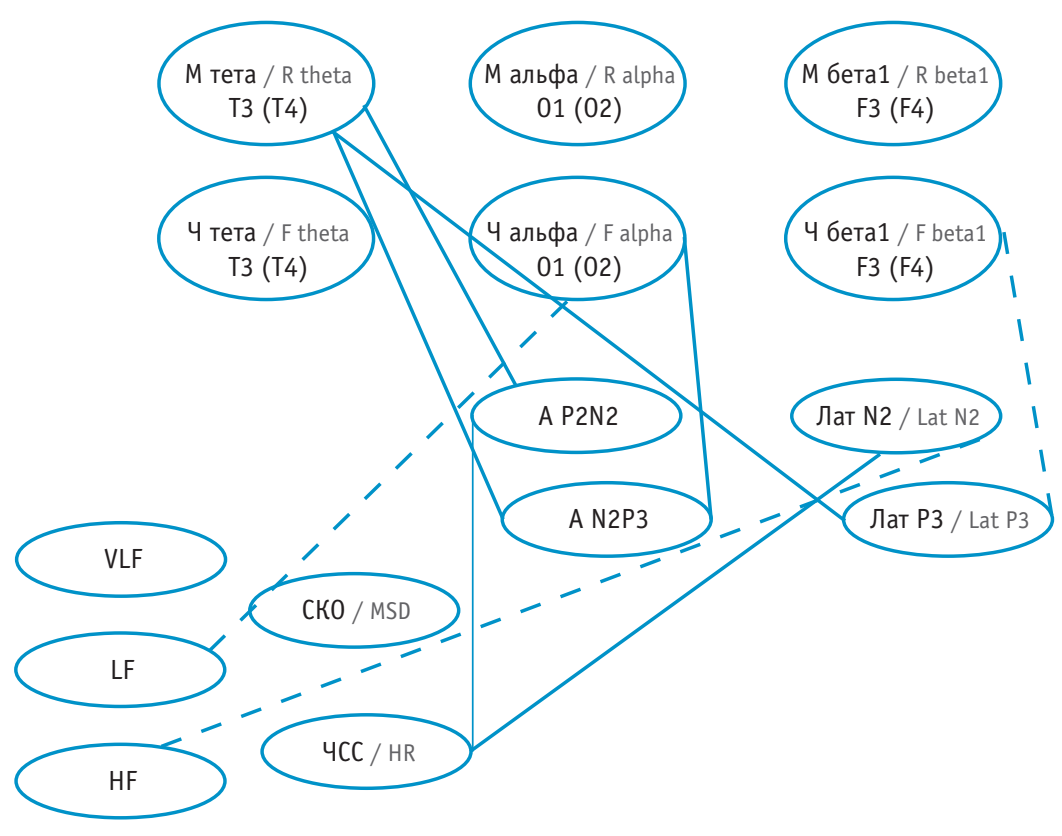

Как следует из представленных графических моделей в виде корреляционных плеяд, у пациентов группы летального исхода определяется увеличение числа линейных корреляций по сравнению с таковым у больных с благоприятным прогнозом. Данные феномены отражают увеличение сопряжения в функционировании физиологических механизмов и, следовательно, ограничение функциональных резервов в этой группе.

\section{ЗАКЛЮЧЕНИЕ}

Неоднородность прогноза острого периода геморрагического инсульта имеет определенные физиологические корреляты. Летальному исходу предшествуют редукция основного коркового ритма, нарастание медленно-волновой активности, по результатам ЭЭГ, снижение активации механизмов опознания стимула, по данным когнитивных вызванных потенциалов Р300.

Анализ степени сопряжения физиологических механизмов регуляции уровня функциональной активности головного мозга (ЭЭГ), нейрофизиологических коррелятов опознания стимула и принятия решения по отношению к нему (Р300), а также механизмов вегетативной регуляции (вариабельности сердечного ритма) указывает на ограничение функциональных резервов у больных с летальным исходом заболевания. 


\section{ЛИТЕРATYPA / REFERENCES}

1. Bogousslavsky J., Caplan L.R., Dewey H.M. et al. Stroke: selected topics. World Federation of Neurology. Seminars in clinical neurology. New York: Demos Medical; 2007. 528 p.

2. Benjamin E.J., Virani S.S., Callaway C.W. et al. Heart disease and stroke statistics - 2018 update. A report from the American Heart Association. Circulation. 2018; 137: e67-492. D0I: 10.1161/ CIR.0000000000000558

3. de Oliveira Manoel A.L., Goffi A., Zampieri F.G. et al. The critical care management of spontaneous intracranial hemorrhage: a contemporary review. Crit. Care. 2016; 20: 272. D0I: 10.1186/ s13054-016-1432-0

4. Черний В.И., Андронова И.А., Городник Г.А. и др. Исследование ЭЭГ-предикторов нейромедиаторной и нейроглиальной активности у больных с острой черебральной недостаточностью различного генеза. Медицина неотложных состояний. 2016; 4(75): 45-56. [Cherniy V.I., Andronova I.A., Gorodnik G.A. et al. Evaluation of EEG predictors of neurally-mediated and neurogliac activity in patients with acute cerebral insufficiency of various genesis. Emergency Medicine. 2016; 4(75): 45-56. (in Russian)]

5. Glushakova O.Y., Glushakov A.V., Miller E.R. et al. Biomarkers for acute diagnosis and management of stroke in neurointensive care units. Brain Circ. 2016; 2(1): 28-47. DOI: 10.4103/23948108.178546

6. Казанцев А.Н., Черных К.П., Заркуа Н.Э. и др. Ближайшие и отдаленные результаты каротидной эндартерэктомии в разные периоды ишемического инсульта. Российский медико-биологический вестник имени академика И.П. Павлова. 2020; 28(3): 312-22. [Kazantsev A.N., Chernykh K.P., Zarkua N.E. et al. Immediate and long-term results of carotid endarterectomy in different periods of ischemic stroke. I.P. Pavlov Russian Medical Biological Herald. 2020; 28(3): 312-22. (in Russian)]. DOI: 10.23888/PAVLOVJ2020283312-322
7. Delle-Vigne D., Kornreich C., Verbanck P. et al. The P300 component wave reveals differences in subclinical anxious-depressive states during bimodal oddball tasks: an effect of stimulus congruence. Clin. Neurophysiol. 2015; 126(11): 2108-23. D0I: 10.1016/j. clinph.2015.01.012

8. Chen L., Zhou Y., Liu L. et al. Cortical event-related potentials in Alzheimer's disease and frontotemporal lobar degeneration. J. Neurol. Sci. 2015; 359(1-2): 88-93. D0I: 10.1016/j. jns.2015.10.040

9. Kaplan P.W., Rossetti A.O. EEG patterns and imaging correlations in encephalopathy: encephalopathy part II. J. Clin. Neurophysiol. 2011; 28(3): 233-51. D0I: 10.1097/WNP.0b013e31821c33a0

10. Меркулова М.А., Лапкин М.М., Зорин Р.А. Использование кластерного анализа и теории искусственных нейронных сетей для прогнозирования результативности целенаправленной деятельности человека. Наука молодых (Eruditio Juvenium). 2018; 6(3): 374-82. [Merkulova M.A., Lapkin M.M., Zorin R.A. The use of cluster analysis and the theory of artificial neural networks to predict the effectiveness of targeted human activite. Nauka Molodykh (EruditioJuvenium). 2018; 6(3): 374-82. (in Russian)]. DOI: 10.23888/HMJ201863374-382

11. Koenig J., Thayer J.F. Sex differences in healthy human heart rate variability: a meta-analysis. Neurosci. Biobehav.l Rev. 2016; 64: 288-310. DOI: 10.1016/j.neubiorev.2016.03.007

12. Hénon H., Pasquier F., Leys D. Poststroke dementia. Cerebrovasc. Dis. 2006; 22(1): 61-70. DOI: 10.1159/000092923

13. Джос Ю.С., Калинина Л.П. Когнитивные вызванные потенциалы в нейрофизиологических исследованиях (обзор). Журнал медико-биологических исследований. 2018; 6(3): 223-35. [Dzhos Yu.S., Kalinina L.P. Cognitive event-related potentials in neurophysiology research (review). Journal of Medical and Biological Research. 2018; 6(3): 223-35. (in Russian)]. DOI: 10.17238/ issn2542-1298.2018.6.3.223 D

\section{ЧИТАЙТЕ} нa journaldoctor.ru

инсульт

статьи схожей тематики в выпусках «Доктор.Ру» Неврология Психиатрия:

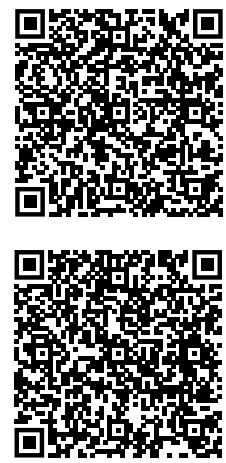

Григорьева В.Н., Сорокина Т.А. Анозогнозия двигательного и когнитивного дефицита в клинике ишемического инсульта: обзор литературы. Доктор.Ру. 2020; 19(9): 33-38. DOI: 10.31550/1727-2378-2020-19-9-33-38

Ястребцева И.П., Кривоногов В.А., Пануева Н.Н., Белова В.В., Бочкова Е.А., Дерябкина Л.Ю. Нейрометаболическая поддержка у пациентов с двигательными нарушениями при инсульте на втором этапе реабилитации. Доктор.Ру. 2020; 19(9): 27-32. DOI: 10.31550/1727-2378-2020-19-9-27-32

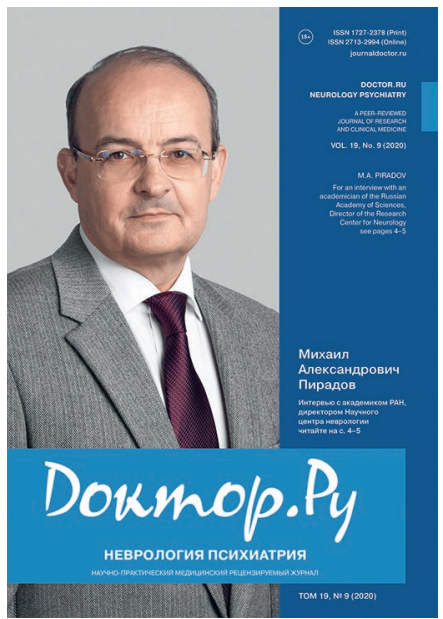

